Global Journal of Pure and Applied Mathematics.

ISSN 0973-1768 Volume 17, Number 2 (2021), pp. 209-213

(C) Research India Publications

https://dx.doi.org/10.37622/GJPAM/17.2.2021.209-213

\title{
Why do we use only multiplicative group in the formation of group ring $R[G]$ ?
}

\author{
Dr. Hiteshwar Singh \\ Retd. Assistant Professor \\ Deptt. of Mathematics, M.L.S.M. \\ College, Darbhanga
}

\author{
Dhananjay Kumar Mishra \\ Univ. Deptt. of Mathematics, \\ L.N.M.U. Darbhanga
}

\begin{abstract}
This research paper is based on the concept of algebraic structure group ring $R[G]$. A group ring structure only includes group $G$ which is closed under multiplication. In group ring structure, we will choose only multiplicative group $G$ over ring $R$. This research paper will provide an information, why do we take a multiplicative group $G$, only to construct an algebraic structure group ring $R[G]$. This paper also clears the concept of group $\operatorname{ring} R[G]$ when group $G$ will be a finite cyclic and infinite cyclic. When we create a group ring $R[G]$ in which group $G$ is infinite as well as cyclic then the given algebraic structure lies between the ring of polynomials and field of rational numbers. If the ring of polynomial will be $K\left[t_{1}, t_{2}, \ldots . t_{n}\right]$ and field of rational $K\left(t_{1}, t_{2}, \ldots . t_{n}\right)$ then we have,

$$
K\left[t_{1}, t_{2}, \ldots . t_{n}\right] \subseteq K[G] \subseteq K\left(t_{1}, t_{2}, \ldots . . t_{n}\right)
$$

Key words:-Group ring, cyclic group, polynomial ring, field of rationals, multiplicative group, infinite cyclic group.
\end{abstract}

1. Introduction: As we know that every group ring $R[G]$ is the set of all elements of the form $\sum_{x \in G} a_{x} \cdot x$, here, $a_{x} \in R$ and $x \in G$. So we can write

$$
R[G]=\left\{\sum_{x \in G} a_{x} \cdot x \mid x \in G \wedge a_{x} \in R\right\} \text {. When we construct a group ring, we take }
$$

only multiplicative group $G$. Why do we choose a multiplicative group, whenever we form a group ring structure? In this paper we have given the answer of this question. 
The structure group ring $R[G]$ is an algebraic structure which depends on the nature of group $G$. In this research paper we have discussed on two types of group $G$.

(i) Finite cyclic group if $\left(\alpha \in K[G], \alpha=\sum_{\tau=0}^{n-1} a_{i} x^{i}\right)$

(ii) Infinite cyclic group or a product of infinite cyclic groups, if $\left(\alpha \in K[G], \quad \alpha=\sum_{-\infty}^{\infty} a_{i} x^{i}\right)$

When group $G$ is a product of infinite cyclic groups then group ring $K[G]$ over field $K$ lies between ring of polynomials over field $K$ and field of rationals. Thus in this paper we have tried to present a group ring when group $G$ is an infinite group.

\section{Why do we use a multiplicative group $G$ in constructing group ring structure $R[G]$ ?}

Group ring $R[G]$ is a set of all formal finite sums of the form, $\alpha=\sum_{g \in G} a_{g} \cdot g$, here $a_{g} \in R$ and finite numbers of $a_{\mathrm{g}}$ are non-zero. In $R[G]$ group $G$ is taken as a multiplicative infinite or finite group. Therefore, we add two different elements $\alpha$ and $\beta$ of $R[G]$, in which group elements remain same but elements of $R$ are chosen as different.

For example,

$$
\alpha+\beta=\left(\sum_{g \in G} a_{g} \cdot g\right)+\left(\sum_{g \in G} b_{g} \cdot g\right)=\sum_{g \in G}\left(a_{g}+b_{g}\right) \cdot g
$$

While in multiplication of two different elements of $R[G]$ we can choose two different elements of group $G$ with different elements of ring $R$.

For example, let us take two different elements $\alpha$ and $\beta$ of group ring $R[G]$ as,

$$
\begin{aligned}
& \alpha \beta=\left(\sum_{g \in G} a_{g} \cdot g\right)\left(\sum_{h \in G} b_{h} \cdot h\right) \\
& =\sum_{g, h \in G}\left(a_{g} b_{h}\right) \cdot(g h) \\
& \gamma=\sum_{z \in G} c_{z} \cdot z, \quad \gamma \text { is the element of } R[G] \\
& c_{z}=a_{g} b_{h} \in R \text { and } z=g h \in G . \text { Hence only multiplicative groups are taken to }
\end{aligned}
$$
construct group ring structure $R[G]$. We cannot add two different elements of $R[G]$ 
which are chosen as

$$
\alpha=\sum_{g \in G} a_{g} \cdot g \text { and } \beta=\sum_{h \in G} b_{h} \cdot h \text {, because here two elements of group } G \text { are } g
$$

and $h$ and $g \neq h$. This means $\sum_{g \in G} a_{g} \cdot g+\sum_{h \in G} b_{h} \cdot h \notin R[G]$ provided that $g \neq h$ or $g$ and $h$ are different, linearly situated vectors. When we take $\operatorname{ring} R$ as a field $K$ and new group ring structure will be $K[G]$. As we can not add two differently situated vectors because group ring $R[G]$ is a set of all same linearly situated vectors. So if we add two differently situated vectors then the resultant can not be the element of same group ring set. But multiplication of two differently situated linear vectors is possible in a group ring $R[G]$. As we have shown in the product of two $R[G]$ elements above. Therefore we will use only multiplicative group in the construction of a group ring structure $R[G]$.

\section{Very interesting examples of group ring $K[G]$ in finite cyclic group $G$ as well as in infinite cyclic group $G$.}

(i) Let there be a finite cyclic group $G=\langle y\rangle$. If order of group $G$ be $n$ means $|G|=n$. The basis of $K[G]$ will be $1, y, y^{2}, \ldots . ., y^{\text {n-1 }}$. Now the each element of $K[G]$ will be uniquely written as, $\alpha=\sum_{i=0}^{n-1} a_{i} \cdot x^{i}$. We choose a polynomial ring $K[t]$ and a natural map $K[t] \rightarrow K[G]$. This will be an epimorphism (surjective). The kernel of this map will be $\left(1-\mathrm{t}^{\mathrm{n}}\right)$. It will be the principal ideal and this domain will be generated by $\left(1-\mathrm{t}^{\mathrm{n}}\right)$. This every polynomial of $K[t]$ will be written as, $p(t) \in K[t], \quad p(t)=\left(1-t^{n}\right) \cdot q(t)$. Therefore from the first law of isomorphism of ring, we have, as $K[G] \cong K[t] /\left(1-t^{n}\right)$.

(ii) But if group $G=\langle y\rangle$ is infinite cyclic. The elements of group ring $K[G]$ will be written uniquely as finite sums of the form $\sum_{-\infty}^{\infty} a_{i} \cdot x^{i}$. There will be a natural mapping $K[t] \rightarrow K[G]$. This time mapping will not an onto mapping. Thus $K[G]$ will be contained isomorphically between $K[t]$ and the rational function field $K(t)$.

4. Lemma : Let us suppose that $G$ be a group which is torsion-free abelian and infinite of order $n$. Then group ring $K[G]$ will be between the polynomial ring and its rational function field. If we suppose a group $G$ as

$$
G=\left\langle y_{1}\right\rangle \times\left\langle y_{2}\right\rangle \times\left\langle y_{3}\right\rangle \ldots \ldots \ldots \times\left\langle y_{n}\right\rangle \text { a direct product of } \mathrm{n} \text { infinite cyclic groups. }
$$

Then we have ring of polynomial

$K\left[t_{1}, t_{2}, t_{3}, \ldots \ldots, t_{\mathrm{n}}\right]$ which will be naturally mapped to $K[G]$ that is

$\phi\left(K\left[t_{1}, t_{2}, t_{3}, \ldots \ldots, t_{\mathrm{n}}\right]\right) \rightarrow K[G]$. We get here

$K\left[t_{1}, t_{2}, t_{3}, \ldots \ldots, t_{\mathrm{n}}\right] \subseteq K[G]$ 
Similarly we have rational field function

$$
\begin{aligned}
& K\left(t_{1}, t_{2}, t_{3}, \ldots \ldots, t_{\mathrm{n}}\right) \text { and we get } \\
& K[G] \subseteq \mathrm{K}\left(t_{1}, t_{2}, t_{3}, \ldots \ldots, t_{\mathrm{n}}\right) . \text { Therefore we obtain, } \\
& K\left[t_{1}, t_{2}, t_{3}, \ldots \ldots, t_{\mathrm{n}}\right] \subseteq K[G] \subseteq K\left(t_{1}, t_{2}, t_{3}, \ldots \ldots, t_{\mathrm{n}}\right) .
\end{aligned}
$$

\section{Proof :}

From the fundamental theorem of abelian groups, any finitely generated torsionfree abelian group $G$ will be a direct product of $\mathrm{n}$ infinite cyclic groups for some integer $n$.

So, we write $G=\left\langle y_{1}\right\rangle \times\left\langle y_{2}\right\rangle \times\left\langle y_{3}\right\rangle \ldots \ldots \ldots \times\left\langle y_{n}\right\rangle$ and each element of group $G$ will be written as uniquely in the form, $y_{1}^{m_{1}} \cdot y_{2}^{m_{2}} \ldots \ldots \ldots . . . y_{n}^{m_{n}}$. There is a natural homorphism $K\left[t_{1}, t_{2}, t_{3}, \ldots, t_{\mathrm{n}}\right] \rightarrow K[G]$ and $t_{\mathrm{i}} \rightarrow y_{\mathrm{i}}$ is an embedding in $K[G]$. Hence we have $K\left[t_{1}, t_{2}, t_{3}, \ldots \ldots, t_{\mathrm{n}}\right] \subseteq K[G]$

Now we choose any element $\alpha \in K[G]$ then for any sufficiently large integer m, we get as, $\left(t_{1}, t_{2}, t_{3}, \ldots \ldots, t_{\mathrm{n}}\right) \cdot \alpha \in K\left[t_{1}, t_{2}, t_{3}, \ldots \ldots, t_{\mathrm{n}}\right]$.

Thus we can say that $K[G]$ is an integral domain and that is why $K[G]$ is contained in the field of rationals. Therefore

$$
\begin{aligned}
& K[G] \subseteq K\left(t_{1}, t_{2}, t_{3}, \ldots \ldots, t_{\mathrm{n}}\right), \text { and finally } \\
& K\left[t_{1}, t_{2}, t_{3}, \ldots ., t_{\mathrm{n}}\right] \subseteq K[G] \subseteq K\left(t_{1}, t_{2}, t_{3}, \ldots ., t_{\mathrm{n}}\right) .
\end{aligned}
$$

\section{Kernel of epimorphism $f: K[t] \rightarrow K[G]$ is a principal ideal generated by $\left(t^{\mathrm{n}}-1\right)$}

As we know that $1 \in K$, so we have $1 . x \in K[G]$. Thus we have obtained that $G \subseteq K[G]$. But it is clear that in group ring $K[G]$ group $G$ is regarded as a multiplicative group. So $1 \in K$ (identity element) and $1 \in K[G]$ as the identity element in $K[G]$, because $G \subseteq$ $K[G]$. If group $G$ is of finite order cyclic group and $|G|=n$ then the elements of group $G$ will be $1, x, x^{2}, x^{3}, \ldots x^{\mathrm{n}-1}$. Thus the identity element of $G$ will be written as $x^{\mathrm{n}}=1$. Therefore, the kernel of an epimorphism $K[t] \rightarrow K[G]$ will be $\left(1-t^{\mathrm{n}}\right)$, which will map $x^{\mathrm{n}}$ $\in K[G]$ in $K[G]$. As we know that $x^{\mathrm{n}}=1, x^{\mathrm{n}} \in G \subseteq K[G]$, so $x^{\mathrm{n}} \in K[G]$ that is an identity element of $K[G]$. That is why $\left(1-t^{\mathrm{n}}\right)$ will be kernel of an epimorphism $f, f:(K[t])$ $\rightarrow K[G]$. Thus we will find $f:\left(t^{\mathrm{n}}-1\right) \rightarrow 1 \in K[G]$. In other words the kernel of epimorphism $f$ will be the principal ideal generated by $\left(t^{\mathrm{n}}-1\right)$.

\section{Conclusions:}

In this research paper we have found that whenever we construct a group ring structure $R[G]$, we will have to take, only multiplicative group $G$. This means in every group ring structure the group $G$ will be closed under multiplication operation. Secondly we have also get that in a group ring $K[G]$ if group $G$ is a finite cyclic then, $K[G] \cong K[t] /\left(1-t^{\mathrm{n}}\right)$. 
Here $K[t]$ is a ring of all polynomials in indeterminate $t$. Thirdly we have found that if group $G$ is infinite cyclic and torsion-free then group ring formed by taking this group will lie between the ring of polynomial and the field of rationals. It can be expressed as, $K\left[t_{1}, t_{2}, t_{3}, \ldots . ., t_{\mathrm{n}}\right] \subseteq K[G] \subseteq K\left(t_{1}, t_{2}, t_{3}, \ldots . ., t_{\mathrm{n}}\right)$.

or ring of polynomial (over field $K) \subseteq K[G] \subseteq$ field of rationals.

\section{Acknowledgement :}

This research paper has been prepared by second author during his Ph.D. research work.

\section{References:}

1 D.S. Passman, The Algebraic Structure of Group Rings. Dover Publication, Inc., Mineola, New York, 2011.

2. Hazewinkel, Michiel, ed. (2001) [1994], "Cyclic group", Encyclopedia of Mathematics, Springer Science + Business Media B.V./Klumer Academic Publishers, ISBN 978-1-55608-010-4.

3. Herstein p. 153.

4. Herstein, Hall, p. 73.

5 Weisstein, Eric W. "Field of Rationals".

6. Michiel Hazewinkel, Nadiya Gubareni, Nadezhda Mikhalovna Gubareni, Vladimir V. Kirichenko. Algebras, rings and modules. Volume 1, 2004. Springer, 2004. ISBN 1-4020-2690-0.

7. Jacobson, Nathan (2009). Basic algebra, 2 ( $2^{\text {nd }}$ ed), Dover, ISBN 978-0-48647187-7.

8. D.S. Passman, The algebraic structure of group ring. Wiley (1977). 
